\title{
Routing Protocol of Cognitive Ad Hoc Network
}

\author{
Chunxia Wang \\ Department Of Computer and Information Technology, Shangqiu Normal College, \\ Henan Shangqiu, 476000, China \\ ch.x.wang@163.com
}

\begin{abstract}
This paper proposes a priori cross layer routing protocol CLC-DSDV routing protocol in cognitive Ad Hoc network, the protocol will combine channel management mechanism and DSDV routing protocol in Ad Hoc network, and aiming at the existing problem of multi channel DSDV-MC routing protocol such as primary user interference, routing load imbalance, routing reconfiguration have been improved, in order to achieve the effective use of wireless spectrum of cognitive Ad Hoc networks. Concrete improvements on the routing protocol are described: joining the node load considerations in the routing process; through the information of the global routing table of each node for distribution in channel allocation; channel allocation information embedded in routing newspaper information and broadcasting timely; starting routing update packets to ensure compliance with the neighbor node information synchronization at the channel switching time. The simulation of the routing protocol based on NS2, the simulation results show that the CLC-DSDV routing protocol can effectively improve the utilization of licensed spectrum rate, throughput, delay, packet loss rate, routing load and other aspects can be more outstanding than the original multi channel routing protocol DSDV-MC.
\end{abstract}

Keywords: Cognitive Radio; Ad Hoc Network; CLC-DSDV; Routing Protocol

\section{Introduction}

In recent years, with the large-scale expansion of wireless network, independent of fixed infrastructure self-organizing wireless Ad Hoc network technology has obtained the very rapid development. At the same time, as the user node function of Ad Hoc network increased gradually, the applications are more and more extensive, but at the same time, communication frequency band wireless communication is also becoming more and more crowded, the problem of frequency resource shortage. Although Ad Hoc network support for multiple wireless protocols, but the frequency of communication application of the existing communication technology concentrated more on the $900 \mathrm{MHZ}$ and $2.4 \mathrm{GHZ}$ industrial, scientific and medical band.

With the massive expansion of wireless networks, communication frequency band where they are becoming more and more crowded. At the same time, in some frequency bands such as $400 \mathrm{MHZ}$ to $700 \mathrm{MHZ}$, frequency band utilization rate is far lower than the normal value. According to the America Federal Communications Commission data, spectrum allocation and utilization range of variation in space and time is from $15 \%$ to $85 \%$. Another report showed that, even in the crowded area, there are more than $70 \%$ of the allocated spectrum have been used. Now the existence of spectral partitioning problem is caused due to historical reasons, long-term, and spectrum allocation but also difference in geographic scope, and this has led to the spectrum resources and low utilization ratio imbalance. In order to improve the spectrum utilization rate, to solve the spectrum scarcity problem, the 
existing research mainly adopt three kinds of methods: one is based on the existing wireless network with link adaptation technology, modulation and coding of advanced technology and multi antenna technology; the second category is the coexistence of a variety of wireless transmission technology of communication system, such as ultra wideband system work at the frequency of $3.10 \mathrm{GHz}$ and traditional narrowband systems coexist techniques, but these techniques to improve the spectrum utilization efficiency while increasing the interference limits the capacity of the communication system and flexibility. The third category is the cognitive radio technology. In the three methods of cognitive radio technology is considered to be the most suitable for outdoor communication technology. The proposed cognitive radio technology to solve the problem of the shortage of spectrum resources this thorny problem. The technology can be understood as a dynamic spectrum allocation technology in terms of spectral distribution. The concept of cognitive radio was first proposed by Dr. Joseph Mitola in 1999, which is mainly to solve the problem of spectrum resource scarcity, the basic idea is to improve the utilization of available spectrum. The basic starting point of cognitive radio is: in order to improve the spectrum utilization, with cognitive function unlicensed users (secondary users) can be in the authorized user has licensed spectrum (primary users) without or communication service only a few cases in accordance with certain "opportunistic (Opportunistic Way)" the way to work in the licensed frequency band within this spectrum resources appear in the space domain, time domain and in frequency domain can be used to be called "spectrum holes"). The core idea of cognitive radio is to make the wireless communication device has found "spectrum holes" and to achieve "spectrum sharing" premise does not affect the main user communication, the final completion of the wireless communication process.

Once the cognitive network system discovery that it can find appropriate "spectrum holes" for communication, cognitive network will carry on the wireless communication packets on the upper application. To find the appropriate "spectrum holes", the mobile terminal needs perceived in advance communication environment (such as the spectrum hole, geographical location, available wireless system or network, suitable type of service), and in view of the user characteristics and needs analysis, and then reconfigured to the case of adaptive change of system parameters on its. For example, when the cognitive mobile terminal sense there is the presence of the WiFi network and GSM network in the surrounding environment, and digital TV band surrounding the existence of spectrum holes, the cognitive wireless terminal may decide to download files via WiFi network, a phone call is made through the GSM network, and communicate through the spectrum holes and other cognitive users. Cognitive terminal can negotiate with other cognitive users to determine the effective communication frequency in order to achieve communication. The negotiation process may be through wireless networks are the underlying infrastructure may also be done through an Ad Hoc network.

Cognitive radio network with the traditional wireless network has a significant difference, the latter is assigned using immobilized spectrum, thus causing a spectrum utilization rate reduce. The cognitive radio network is dynamic spectrum allocation for wireless applications in the surrounding environment and dynamic adjustment of the communication parameters (such as the communication carrier frequency channel transmit power). When the main user (authorized users) exist, user cognitive network must unconditionally release spectrum. That is to say, in cognitive radio network, we must guarantee the communication not cognitive users generate communication interference to primary users. 
Cognitive radio technology as the free use of spectrum resources technology, in the Ad Hoc network application is very appropriate, especially suitable for the Ad Hoc cognitive radio emergency network. However, when the application of cognitive radio technology in low power multi hop Ad Hoc networks, because of its multi hop communication, dynamic network topology changes, the spectrum state with time and physical location changes and other characteristics, to realize the system needs a new MAC protocol and the new routing protocol. Generally speaking, communication between cognitive users need to use a shared transmission control channel to complete the routing and signaling control information in one or more of the. In cognitive Ad Hoc network technology research in an important characteristic is, meet all kinds of the solution of the problem must be considered together, the design process in the multi-layer protocol in which the cognitive radio technology, routing technology is the key technology of dynamic spectrum access in cognitive scene in Ad Hoc network.

Similar to general cognitive network, Ad Hoc network is composed of cognitive secondary users (i.e., non authorized users, the cognitive user) composition, spectrum resources in the secondary users, the primary user (i.e., authorized users) can always deprivation, the occupation of the corresponding spectrum, which leads to the available channel resources of the whole of the cognitive network with time, spatial variation. Cognitive radio system can be divided into three functional modules: cognitive modules (perceived environmental parameters), self organizing module (analysis of perceived information), reconfiguration module (adaptive adjustment of the wireless resources).

\section{Related Work}

The characteristics of Ad Hoc networks have not infrastructure, the mobile node in Ad Hoc network can be directly with the neighbor node (node receives its emission range) the connection, the overall mobile nodes form a network. Establish the communication between different nodes can be connected through different communication technologies. Ad Hoc network is a special kind of wireless mobile network, due to the wireless channel, the limited power, distributed control technology, it is more vulnerable to eavesdropping, active intrusion, denial of service, deprived of "sleep" and other network attacks. In addition, Ad Hoc network is composed of nodes act as routers themselves, not the existence of naming server and directory server and other network facilities, also does not exist the concept of network boundary. This makes the security problem of Ad Hoc network is very complex, the traditional network of many security policies and mechanisms will no longer apply. Therefore, channel encryption, anti disturbance, user authentication, key management, access control and other safety measures need special consideration. Cognitive radio technology as the free use of spectrum resources technology, in the Ad Hoc network application is very appropriate, especially suitable for the Ad Hoc cognitive radio emergency network. However, when the application of cognitive radio technology in low power multi hop Ad Hoc network, to realize the system needs a new MAC protocol and the new routing protocol. Generally speaking, communication between cognitive users need to use a shared transmission control channel to complete the routing and signaling control information in one or more than one, so the design of routing protocol and MAC protocol need to consider the requirements of control channel. Cognitive radio technology because it can make full use of existing spectrum resources in wireless networks, since the application foreground is very good, but at the same time, due to the power limitation, wireless network itself, node mobility, topology and 
changeable, characteristics of complex routing establishment way, need to combine the characteristics of cognitive radio routing protocol and the improved MAC protocol.

Compared with the traditional Ad Hoc network, one of the most important difference of cognitive Ad Hoc networks is the spectrum resources cognitive in $\mathrm{Ad}$ Hoc network will produce a corresponding change as the main user are busy or idle state. This is because the primary user (for spectrum is authorized user) for spectrum access than cognitive users with the use of a higher priority. In cognitive Ad Hoc network, the available communication spectrum may be as great changes have taken place in space and time. This means that changes in each cognitive user will be with the primary user activity and the occurrence of spectrum resources. In contrast, ordinary Ad Hoc network are usually in a communication channel pre-defined (can be understood as the corresponding spectrum) on communication, of course, the common Ad Hoc users in a network is also possible from the spectrum in a series by selecting a channel of a fixed mechanism, but the selection process is not dependent on the main users of the busy activity. In other words, cognitive Ad Hoc network and common Hoc network Ad biggest difference lies in whether or not to consider the presence of primary user.

Due to the absence of a wireless access point AP, the Ad Hoc network itself is lack of centralized control, so it must be realized through collaborative broadcasting certain topology information collection. In the ordinary Ad Hoc network, this process can be adopted in periodic broadcast channel to realize. In the cognitive Ad Hoc networks, due to the licensed spectrum (i.e., the available spectrum) spectrum above the general exist in large range, so in all likelihood broadcast topology information channel is not realistic. Therefore, the cognitive Ad Hoc network is more likely to occur in a topology information collection is not complete, which leads to conflict between cognitive user generated interference or different cogniti ve users to the primary users.

Because the available spectrum resources and the uncertainty of the wide range of spectrum resources, compared to the general Ad Hoc network, a handover to a spectrum are more prone to multi hop communication process in the end of the cognitive Ad Hoc network in the end, this is because of the presence of primary user will cause the available spectrum resources in time and space on the uncertainty. Therefore, cognitive processes and spectrum allocation Ad Hoc network routing protocols need collaborative process, to minimize unnecessary switching channel. At the same time, the cognitive Ad Hoc network in the end to end communication quality control involves not only the business flow, also relates to the number of available channels and all possible activities of channel spectrum, primary user (process based on spectrum sensing mentioned) etc..

In the general Ad Hoc network, routing may route invalid path impassability due to node movement, this can be through the next hop node ultra limit number does not respond to information to be found. In the cognitive Ad Hoc network, even if the node does not move, because the occupation of channel of primary user, also can cause the path cannot be communication. Therefore, in the selection of communication path has not, shall determine whether the resulting due to node mobility or spectrum resource mobility, and then take the recovery strategy for certain security communication. We will in the following chapters discuss in detail the channel allocation of cognitive Ad Hoc network and routing.

Ad Hoc network has no center, independent, limited resources and other characteristics, so the cognitive technology if applied to low power multi hop Ad Hoc network also need spectrum to design new MAC protocol and routing protocol 
to support distributed sharing. The existing cognitive radio circuit is composed of three point agreement roughly according to classification:

1) Spectrum decision whether or not to engage with the routing process, such as the integrated spectrum availability and comprehensive selection of a node routing;

2) During route establishment whether considering the main user busy information, that is to say the secondary users (cognitive user) is in the routing process can get the main user busy to avoid the interference;

3) The selection process is in the routing protocol considers spectrum decision and reconfiguration process, routing establishment if band change, and choose another path.

Based on these three points, can use the existing routing protocols for cognitive wireless network is divided into the following three categories.

(1) The establishment of the spectrum when routing choice, but not for the main user judgment.

Routing protocol in the network layer is responsible for packet forwarding can choose the next hop node, and at the same time, aiming at the communication connection for screening and allocation of the available spectrum. Therefore, spectrum selection and path selection at the same time, it can also through the selection of a plurality of selectable band in the spectrum allocation and makes the routing stability is greatly enhanced. Consisting of cognitive user routing need channel allocated to it as the same, because it can make the spectrum handoff delay in the process of communication is greatly reduced. However, if the will were the same in adjacent channel in a communication connection leads to spectrum sharing (via the aforementioned IEEE802.11MAC protocol, a single channel at the same time only allows a node to collect the bag or contract), which lowers the overall efficiency of the two routes. Single transceiver multi hop cognitive routing protocol is a routing protocol of this type. First, such as the basic AODV routing protocol process, cognitive node to all possible channels to send a route request RREQ, and the RREQ request through all possible channels transmitted to destination nodes. Then according to the shortest path according to the channel switching time, channel estimation and data transmission congestion choice ideal spectrum.

(2) Considering the main user busy channel availability information, routing algorithm. Protection in cognitive network routing must be some way for the ongoing communication or will be the primary users of the. In such cases, the routing must be established to avoid occupying known there would be massive primary user communication area, it makes the routing hops larger in a certain extent, but will cause interference to primary users become smaller.

(3) Routing and spectrum allocation process, routing protocol algorithm combining the reconfiguration process

Compared with the previous two kinds of routing protocol, the difference between third kinds of routing protocol is the most important in the primary user interference can lead to reconfiguration user spectrum is not available for routing, in spectrum affected for switching frequency spectrum to ensure communication as usual or routing are re selected to re offer communication conditions. SPEAR routing protocol requires routing establishment stage will choose a plurality of available routing to ensure routing switch, the protocol guarantees on the same route on the same channel, but also limits the amount of information in the process of sending route establishment in routing. In the process of communication, the destination node choice final routing, each jumping on the corresponding channel will be at this stage to be retained for a preset time. At each node, switching may spectrum in the routing operation process as long as the end-to-end routing can guarantee a certain 
throughput and delay. If the routing adaptive process fails, then the SPEAR routing protocol from the source node to restart a new route establishment process.

\section{Model Descriptions}

Channel allocation of multi channel routing protocol, it is not considered primary user's channel occupancy and channel assignment and channel access cognitive Ad Hoc network must first be the maximum to avoid the normal communication of the primary user interference. Obviously, if the channel allocation mechanism of these routing protocols directly applied in cognitive context, will cause serious interference to primary users, then it is difficult to guarantee the quality of communication. There are two kinds of traditional method to solve the problem of coexistence with the primary users: one time once the user to detect the primary user work cannot use this channel; the other is a secondary user to detect the primary user work but still can use the licensed channel interference, but it produced to the main user must be in the tolerance range. These two different ways each have advantages and disadvantages in different environment. The central node does not exist in a distributed network, and distributed node can grasp information often is limited, if the use of complex protocol interaction and the algorithm will increase causing overhead, drop may instead create performance. In cognitive Ad Hoc network multi channel, if the node cannot be initialized to have common communication channels between nodes, is unable to establish the initial communication, which requires channel has an initialization option agreement, to complete the nodes can be in the same "communication platform", increase the success probability of communication. In addition, there are nodes whether synchronization problem. Channel allocation strategy for distributed cognitive radio is mainly divided into three types, including segmented and channel hopping allocation strategy type require synchronization between the nodes, this kind of synchronization refers to the synchronization of the nodes in the system on time. However, the precision of time synchronization inevitable requirement of nodes to be high. If the node accuracy is not high enough, then between nodes in the whole network collision probability will be increased dramatically, resulting decline increased frame loss rate and network throughput. Even if the node time precision is high enough, in a distributed network, also need to have efficient protocol to coordinate, in the coordination will encounter problems, for example, selecting which nodes as synchronous reference node, this node should be static or dynamic change, synchronization should be global or local. Visible, in the system time synchronization protocol running instability brought. If no such synchronization, then the network will become more flexible, more conducive to new nodes join. But this has created a new problem: how to interaction between the nodes, if the sending and receiving node sensing to channel variations are not synchronized, then communication will be very difficult to build. In order to achieve efficient use of spectrum resources multi channel, must solve the cognitive node initialization and synchronization problem. Channel allocation in the SPEAR routing protocol in, after the end of each channel allocation of cognitive nodes will have 1.5 duplex data transceiver, then, must be the same channel usage time is divided into slots in order to complete the data sending and receiving mode. At this time, requires strict clock synchronization protocol to achieve channel time slot, there is the problem of synchronization channel. This has greatly increased the limit of routing protocol in practical application.

In cognitive Ad Hoc networks, due to spectrum mobility, not timing state primary users change will cause the change of available spectrum is more frequent than general multi channel, under these conditions, channel switching frequently occurs 
more frequently. Under normal circumstances, a node's channel switching frequently problems usually occur in the multi hop network as the channel of forwarding nodes and two route choice in two or two above route and not the same, this time in order to ensure the two party (or parties) in the normal communication, the node will channel switching to frequently.

In order to ensure the timely and effective communication of control information, in multi channel wireless network communication, usually a common control channel set, used for interactive control frame. At the same time, in order to achieve the normal communication realized through interaction, will select data channel suitable for transmission of data. In the research of MAC protocol for cognitive radio networks in most existing, will use a common control channel. In the common control channel, and can be divided into two kinds: exclusive public channel mode and time interval method. Exclusive public channel usually has a new channel an exclusive control channel and data, a node first competition in public channel, competition to node public channel interaction control frame, and select the appropriate data channel, finally the transmission of data in a data channel. The period at the mode of each node must keep synchronous time slot, in each channel of the node was divided into two periods: the allocation of time and data transmission time, each node is assigned a time slot. In order to realize the normal communication, the node will be resolved in their respective data channel time slot, and a front of their respective frequencies are transferred to the time assigns good channel start data transfer when the data transmission period coming. The control channel allocation approach has the advantage of reducing the interference between nodes, but need to keep tight clock synchronization. In the distributed cognitive radio network, control channel slotted defects exist in the following aspects: (1) selection of control channel, if the choice is also authorized channel, then the control channel will be the main users take may. If the user is the main occupation, the cognitive user network cannot continue to work. And if you don't use authorized channel, non authorized frequency band communication application crowded also enable cognitive nodes may at any time by the interference, the transmission distance is also smaller than the TV bands, and frequency adjustment range of a transceiver is too large to performance put forward higher requirements. Therefore, there are still a lot of uncertainty factors using a fixed control channel, it may have a great impact on the performance of cognitive radio networks. (2) in the case of network load is light, the control channel may meet the demand, but in the case of heavy network load, a control channel may be a lot of nodes at the same time a request to send data cannot be processed, so that the network throughput increases along with the load may be decreased because of congestion. Obviously, in the unique environment of cognitive radio, public channel selection and implementation is a problem. There are some documents some don't need a common control channel method. This method is similar to the frequency hopping; hopping technology will be longer than just cycle. In this way, each node jumping in a unified frequency hopping sequences, at each hop period, compete for channel between nodes, nodes of the channel competition to stay in this channel to communication, and other non competition to channel node will jump to the next frequency continue to compete. The same with the slot method, this method also requires strict clock synchronization between the nodes, and we know that demands a very high between the nodes in the precise synchronization of time, if there is a slight deviation is likely to make a substantial decline in overall network performance.

In cognitive Ad Hoc network, in order to overcome the defect caused by spectrum in cognitive network instability characteristics, spectrum management mechanism is all cognitive users through the exchange of state information different from 
neighboring nodes to determine the process based on cooperative communication, routing protocol as the basis for dealing with the communication process in the network layer also need and spectrum information to combine. In other words, we must also consider the lower channel information can really play the advantages of cognitive radio technology in the design process of a routing protocol. On the system, spectrum mobility this property requires a certain communication connection management mechanism to guarantee the communication of upper application protocol in spectrum handoff time continuity, communication is the need to lower the hidden on the upper instability. To overcome the defects caused by the network characteristics, spectrum management mechanism is all cognitive users through the exchange of state information different from neighboring nodes to determine the process based on cooperative communication.

Traditional multi channel DSDV routing protocol such as DSDV-MC is not considered a primary user's channel occupancy, and channel assignment and channel access cognitive Ad Hoc network must first be the maximum to avoid the normal communication of the primary user interference. Obviously, if the channel allocation mechanism of these routing protocols applied directly in the cognitive context, will cause serious interference to primary users, then it is difficult to guarantee the quality of communication. There are two kinds of traditional method to solve the problem of coexistence with the primary users: one time once the user to detect the primary user work cannot use this channel; the other is a secondary user to detect the primary user work but still can use the licensed channel interference, but it produced to the main user must be in the tolerance range. These two different ways each have advantages and disadvantages in different environment. The central node does not exist in a distributed network, and distributed node can grasp information often is limited, if the use of complex protocol interaction and algorithm, will increase causing overhead, drop may instead create performance.

\section{Improved Node Vector Routing Protocol}

In order to ensure the communication of the primary user is not disturbed, before routing and channel time user initialization, secondary users put forward communication request or routing updates, channel state aware, and according to each channel busy channel are classified into two sets: the available channel sets ACS and non available channel set UACS. Channel allocation principle is in the channel assignment and routing mechanism combined, the channel information is embedded into the routing packet. In proactive routing algorithm, since each cognitive nodes will maintain a system within the node information, also makes every node can receive the channel assignment information. In addition, in the routing control joined the transmission channel information can reduce the implementation complexity of channel assignment information, for example 0 is the complexity hypothesis proposed channel assignment algorithm $\left(\mathrm{d}^{\wedge} 2\right)$, the $\mathrm{D}$ is the maximum network depth (maximum number of neighbor nodes that nodes may have the), through adding routing information packet routing each node, computing complexity can be reduced to 0 .

Routing protocol requires that each cognitive nodes must to all neighbor nodes broadcast their routing table. Each node will broadcast data in cognitive sequence number contains broadcasting and routing information destination node as follows: IP address, the number of hops to the destination node, according to the routing information sequence number of the destination node, the data transmission channel, has the corresponding number, routing load. 
To avoid frequent switching channel problems, in the routing protocol in the proposed channel switching frequency threshold, provided certain, a user node channel in unit time switching number exceeds a certain threshold, the user node channel switching is stopped, the channel assignment is triggered, this communication link the user related on the the next node communication channel is set to lock the lock value, value choice will lead to frequent switching from channel two (or more than two) communication channel selection, channel burden small will serve as locking value. At this time, the original selected communication channel diverse routing paths will become the same channel, channel switching frequently problem solved.

Most multicast routing protocol performance in MANETs network is not very good, mainly because of the dynamic environment, mobile node network topology transform arbitrary strong, frequent and periodic. In addition, bandwidth and the power of battery are limited. These combined factors make the dynamic network topology is very challenging.

$$
p=1-\left(1-p_{f}\right)\left(1-p_{r}\right)
$$

For the cognitive radio network, mainly to solve the main problem of two wireless networks: wireless spectrum resource and licensed spectrum dwindling has not been utilized fully. The cognitive radio network to solve these problems, in cognitive radio networks, wireless nodes by using the licensed spectrum users do not fully make use of communication. Most research on cognitive radio network is mostly studied in the lower layer concentrated, mostly physical layer and MAC layer.

$$
s(k)=p^{k-1}(1-p)
$$

However, routing in multi hop cognitive radio network, is a very important problem, which directly affects the performance of the entire network. Routing protocol is different from the traditional Ad-hoc network, in a cognitive radio network routing faces many challenges, including spectrum using dynamic, heterogeneous resources such as different channel and frequency at the same node, each node synchronization problem of different channels.

$$
E T X=\sum_{k-1}^{\infty} k * s(k)=\frac{1}{1-p}
$$

Therefore, the application of the traditional Ad-hoc network routing scheme to cognitive radio networks, will bring very poor throughput, delay and packet loss rate performance. Uncertainty skill even routing is different from the traditional Ad-hoc network.

$$
E T T=E T X \times \frac{S}{B}
$$

However, in the cognitive radio network all nodes, according to the definition of the traditional Ad-hoc network, one or more nodes are inaccessible, because communication is the primary user.

$$
W C E T T=\sum^{n} E T T_{i}
$$

In cognitive radio network, the main route is divided into two categories, one for the single path routing, multipath routing is a class.

$$
X_{j}=\sum_{i+n} E T T_{i}
$$

\section{Simulation Results}

This paper mainly in this simulation, it is established in the following problems, first, all the secondary users constitute a cognitive Ad Hoc network, power 
transmission and their use of the available channel, the received power transmission parameters are the same. Secondly, as suming it totally has $\mathrm{N}$ data channels (and the available spectrum pool including $\mathrm{N}$ channel) and 1 common control channel. While the $\mathrm{N}$ data channels and common control channel have the same bandwidth, no spectral overlap between the transmission of information, each channel will not generate interference to other channel. And each node by default can be obtained prior to the channel state information (i.e., each node has the ability to complete the spectrum sensing process). The control channel has no primary users, mainly used for sending and receiving control information, data channel for communication between secondary users. Third, the assumption that each node configured transceiver 2.5 duplex, i.e., receiving or sending data packets at a given moment can each transceiver, but not at the same time, and no interference between 2.5 duplex transceiver, namely the two transceiver with a node can also send and receive. Finally, the hypothesis in the cognitive system, in addition to primary users and cognitive Ad Hoc users, it does not exist for other types of communication network, in other words, the cognitive user communication when the control channel or a data channel will not be subject to interference from other networks.

For the corresponding protocol implementation using NS2 simulation tools, and we have carried on some simulations. Because the proposed cognitive DSDV routing protocols compared to the channel utilization of two kinds of routing protocol for multi hop environment and single hop environment, then according to two protocols in different contract performance rate of specific situations such as throughput, packet loss rate, routing overhead compared. Finally, according to the CLC-DSDV routing protocol separately simulation analysis of single hop and multi hop environments. Simulation experiments were performed in a single hop network and multi hop network, in a single hop network, topological environment is set to $265 \mathrm{~m} * 265 \mathrm{~m}$, but the node transmission power set is enough to cover all the nodes in the simulation environment, so all the destination nodes in the source node's transmitting and receiving range, every communication need only a jump. In the scene, the number of nodes in each communication set to half the total number of nodes, the node transmission power is set to $265 \mathrm{~m}$. In the simulation hypothesis communication time users must be built on the premise of not affecting the primary users, and we assume that the available spectrum (spectrum holes) is changing the form of exponential distribution, or the main users of the data channel occupancy is subject to exponential distribution, the related parameters were $\lambda_{1}, \lambda_{2}$.

The protocol type of the protocol, node number is 60 , the moving range is $265 \mathrm{~m} * 265 \mathrm{~m}$, each node moving speed is $25 \mathrm{~m} / \mathrm{s}$, the rest time is $55 \mathrm{~s}$, the simulation time is $120 \mathrm{~s}$, the bandwidth of the channel is $2 \mathrm{M}$, the type of business is the video, packet size is $1 \mathrm{~KB}$, the rate is 120 packets/s, the experiment repeated 100 times, exponential distribution $\lambda_{1}=0.4, \lambda_{2}=0.6$. 


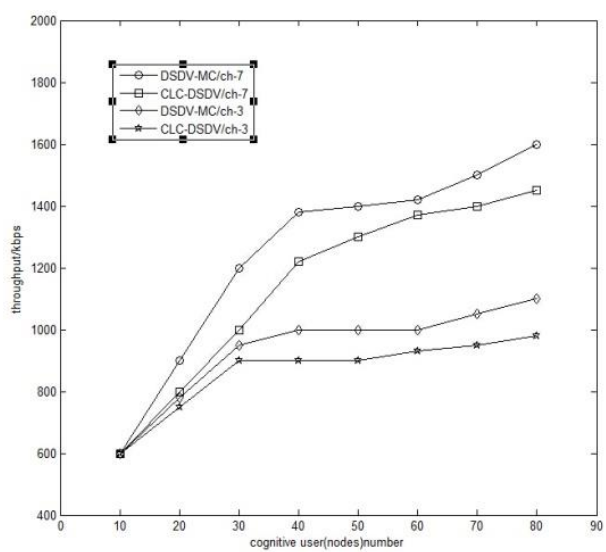

Figure 1. Throughput in Multi Hop Networks Change Chart

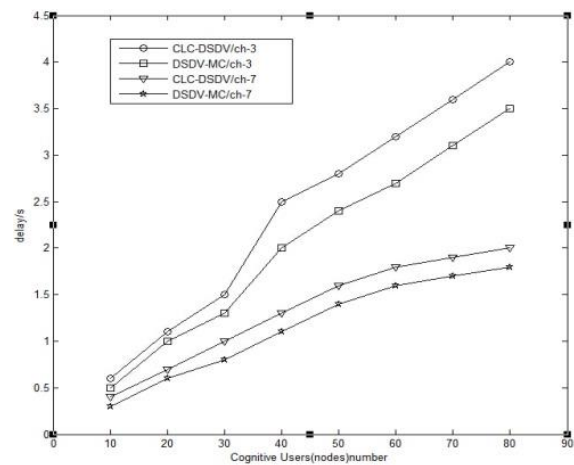

Figure 2. Delay Variation Diagram in Multi Hop Networks

Next, we focus on the CLC-DSDV routing protocol performance system in different cognitive user node number simulation analysis. In this simulation, we studied the routing protocol in multi hop and single hop environment, and compared the data of the two cases. Figure 1 and 2 represent the throughput of CLC-DSDV routing protocols in different scenarios. As can be seen, whether in a single hop or multi hop environment, CLC-DSDV routing protocol can successfully completed in the cognitive scene communication. The reason similar to the previous, the throughput will increase with the number of channels, increased in different degrees, such as can be seen from the graph, the 20 nodes and more cases, the number of channels more system throughput more this is because, the number of channels increases, means that the main user is idle, the more available channel resources. And in the number of nodes (more than 20 nodes in the graph), multiple channel case, the protocol can make full use of spectrum resources. Figure 1 and 2 by comparison, we can see that, when the number of channel and node is same, the throughput of single hop environment is much larger, the reason same to above: multi-channel cognitive scene routing forwarding node communication busy or idle instability and channel complexity decide this point. 


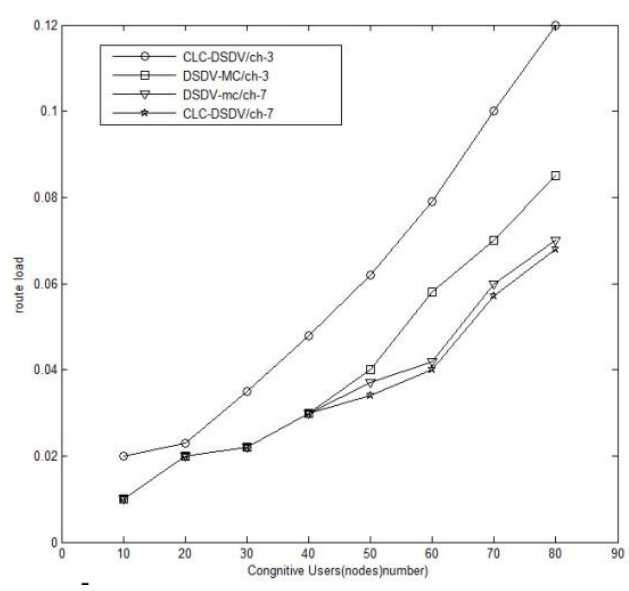

Figure 3. Multi Hop Routing Load Change Chart

\section{Conclusion}

This article in view of the cross-layer routing protocol model, the routing protocol implementation, such as channel allocation, routing packet information, channel switching, routing algorithms and other aspects in detail : adding a node load considerations in the routing process ; the channel allocation performed by the global routing table information of each node; the channel assignment information is embedded in the routing information reported and promptly broadcast ; starting routing update package to ensure that the information is synchronized with the neighbor nodes at the time of channel switching.

\section{Acknowledgments}

This work is financially supported by basic and frontier project of Science and Technology Department of Henan province, China (No 142300410334), the funding scheme for young backbone teachers of colleges and universities in Henan Province, China.

\section{References}

[1] W. B. Heinzelman, A. P. Chandrakasan and H. Balakrishnan, "An Application -Specificprotocol Architecture for Wireless Microsensor Networks", IEEE Trans, on Wireless Communications, vol. 1, (2002) October, pp. 660-670.

[2] S. Cui, A. J. Goldsmith and A. Bahai, "Energy-Efficiency of MIMO And Cooperative MIMO Techniques In Sensor Networks", IEEE Journal on Selected Areas in Communications, vol. 22, (2004), pp. 1089-1098.

[3] S. K. Jayaweera, "Virtual MIMO-based Cooperative Communication forEnergy-constrained Wireless Sensor Networks". IEEE Trans, on Wireless Communications, vol. 5, (2006) May, pp. 984-989.

[4] X. Li, M. Chen and W. Liu, "Application Of STBC-Encoded Cooperative Transmissions in Wireless Sensor Networks”, IEEE Signal Processing Letters, vol. 12, (2005), pp. 134-137.

[5] Y. Yuan, M. Chen and T. Kwon, "A Novel Cluster-Based Cooperative Mimoscheme For Multi-Hop Wireless Sensor Networks", EURASIP Journal on Wireless Communications and Networking, vol. 2006, (2006), pp. 1-9.

[6] A. Paulraj, R. Nabar, and D. Gore, "Introduction to Space-Time Wireless Communications", Cambridge University Press, Cambridge, U.K., (2003).

[7] S. K. Jayaweera, "Energy Analysis Of MIMO Techniques In Wireless Sensor Networks", In Proceedings of the 38th Annual Conference on Information Sciences and Systems (CISS), (2004) March. 
[8] S. K. Jayaweera, "An Energy-Efficient Virtual MIMO Communications Architecture Basedon V-BLAST Processing For Distributed Wireless Sensor Networks", Sensor and Ad Hoc Communications and Networks, 2004 First Annual IEEE Communications Society Conference, (2004), pp. 299-308.

[9] M. Dohler, E. Lefranc and H. Aghvami, "Virtual Antenna Arrays For Future Wirelessmobile Communication Systems", ICT 2002, Beijing, China, (2002) June.

[10] M. Dohler, E. Lefranc and H. Aghvami, "Space-Time Block Codes For Virtual Antennaarrays", PIMRC, Lisbon, Portugal, (2002) September.

[11] S. M. Alamouti, “A Simple Transmit Diversity Technique for Wireless Communications”, IEEE Journ. Select. Areas. Commun. vol. 16, no. 8, (1998) October, pp. 1451-1458.

[12] V. Tarokh, H. Jafarkhani, and A. R. Calderbank, "Space-Time Block Codes From Orthogonal Designs", IEEE Trans. Inf. Theory, vol. 45, no. 5, (1999) July, pp. 1456-1467.

[13] Z. Zhou, S. Zhou, S. Cui and J.-H. Cui, "Energy-Efficient Cooperative Communication in a Clustered Wireless Sensor Network", IEEE Trans, on Vihicular Technology, vol. 57, no. 6, (2008) November, pp. 3618-3628.

[14] X. Li, "Energy Efficient Wireless Sensor Networks with Transmission Diversity", IEEElectronics Letters, vol. 39, (2003) November, pp. 1753-1755.

[15] C. Wenqing and Y. Yong, "Virtual MIMO Protocol Based on Clustering for Wireless Sensor Network", Presented at Proceedings of the 10th IEEE Symposium on Computer Communications, Cartagena, Spain, (2005).

[16] J. N. Laneman and G. W. Womell, "Distributed Space-Time-Coded Protocols For Exploitingcooperative Diversity in Wireless Networks", IEEE Transactions on Information Theory, vol. 49, (2003) October, pp. 2415-2425.

[17] S. Cui and A. Goldsmith, "Cross-Layer Design Of Energy-Constrained Networks Using Cooperative MIMO Techniques”, EURASIP Signal Process, vol. 86, no. 8, (2006) August, pp. 1804-1814.

[18] A. Sendonaris, E. Erkip and B. Aazhang, "User Cooperation Diversity-Part I: Systemdescription”, IEEE Trans. Commun., vol. 51, no. 11, (2003) November, pp. 1927-1938.

[19] Y. C. Liang, K. C. Chen and G. Y. Li, "Cognitive Radio Networking and Communications: An Overview”, IEEE Transactions on Vehicular Technology, vol. 60, no. 7, (2011), pp. 3386-3407.

\section{Authors}

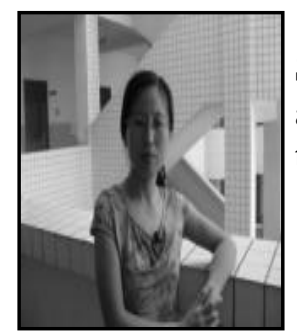

Chunxia Wang received B.Eng and M.Eng Degree in Computer Science and Technology from ZhengZhou University, China in 1997 and 2004 respectively. She is currently researching on Internet of things, Data mining. 
International Journal of Future Generation Communication and Networking Vol. 8, No. 5 (2015) 